\title{
Diabetes and the kidney
}

\author{
Authors: Tahseen A Chowdhury ${ }^{\mathrm{A}}$ and Omer $\mathrm{Ali}^{\mathrm{B}}$
}

Diabetes is the most common cause of end-stage kidney disease. Randomised controlled trials have shown a significant benefit of sodium-glucose transporter-2 inhibitors in patients with diabetic kidney disease (DKD), and guidelines now suggest these drugs should be considered in all patients with DKD irrespective of glucose control. Glucagon-like peptide-1 receptor agonists have shown some benefit in reducing progression of albuminuria in DKD and should also be considered early in the therapeutic pathway. Management of diabetes in patients on renal replacement therapy (dialysis or transplantation) is uniquely challenging. This article outlines guidance on management of glucose in these vulnerable groups of patients.

\section{Introduction}

Diabetes prevalence worldwide, particularly that of type 2 diabetes (T2D) is rapidly increasing. ${ }^{1}$ Diabetes is the most common cause of end-stage renal disease (ESRD) worldwide; it is estimated that around $40 \%$ of people living with T2D have diabetic kidney disease (DKD). ${ }^{2,3}$ The presence of chronic kidney disease (CKD) or albuminuria are independent predictors of cardiovascular morbidity and mortality in people with diabetes. ${ }^{4}$

Here, we review developments in the management of patients with T2D and DKD over recent years. In addition, we discuss the management of hyperglycaemia in patients with diabetes on renal replacement therapy (RRT; dialysis or renal transplantation).

\section{Management of diabetic nephropathy: a new paradigm}

\section{Diagnosis of DKD}

DKD is a clinical diagnosis defined as persistently reduced estimated glomerular filtration rate (eGFR) $<60 \mathrm{~mL} / \mathrm{min} / 1.73 \mathrm{~m}^{2}$ and/or the presence of microalbuminuria (albumin:creatinine ratio (ACR) 3-30 mg/mmol) / macroalbuminuria (ACR $>30 \mathrm{mg} / \mathrm{mmol}$ ) among patients with diabetes. Every person living with diabetes (type 1 diabetes (T1D), T2D and other forms) should undergo at least annual tests of eGFR and ACR, ideally using a first voided urine sample. Elevated ACR should be confirmed with a repeat

Authors: ${ }^{\text {A }}$ consultant in diabetes, The Royal London Hospital,

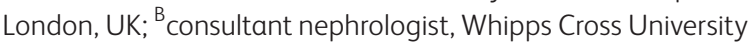
Hospital, London, UK sample, as recent exercise or a high protein meal may elevate ACR in the absence of significant renal disease.

The finding of an elevated ACR in a person with diabetes should elicit further investigation. Not all renal disease in people with diabetes is DKD; therefore, dipstick testing for haematuria and exclusion of urinary tract infection (UTI) is a minimum requirement. Presence of haematuria requires further investigation. Other clues to the possibility of non-diabetes-related kidney disease include the absence of diabetic retinopathy, severe or resistant hypertension, previously normal ACR, rapid worsening of eGFR or the presence of systemic illness. In the absence of these findings, most people with diabetes and elevated ACR will have DKD, and require no further investigation.

\section{Management of DKD}

Management of DKD involves tight control of blood pressure $(<130 / 80 \mathrm{mmHg})$ using angiotensin converting enzyme inhibitors

\section{Key points}

Diabetic kidney disease is an important cause of end-stage renal disease, and early detection and management may prevent or delay development of renal failure.

Prescription of sodium-glucose transporter- 2 inhibitor drugs to people with type 2 diabetes who have evidence of chronic kidney disease or albuminuria should be considered, irrespective of glucose control, and in addition to reninangiotensin system blockade therapy.

Glucagon-like peptide-1 receptor analogues are also useful in diabetic kidney disease and should be considered early in the therapeutic pathway.

For patients with diabetes on haemodialysis, avoidance of hypoglycaemia is paramount. Use of continuous or flash glucose monitoring systems may facilitate adjustment of glucose lowering therapy.

Post-transplant diabetes mellitus is an important clinical entity, and requires active screening and management.

KEYWORDS: diabetes, nephropathy, sodium-glucose transporter-2, dialysis, transplantation

DOI: 10.7861/clinmed.2021-0144 
(ACEI) or angiotensin receptor blockers (ARB) as a first-line therapy. ${ }^{5}$ Even in the absence of hypertension, ACEI or ARB therapy should be offered, to maximum tolerated dose, with advice for avoidance of pregnancy in women of child bearing age. Patients with DKD are frequently multi-morbid, often living with a number of long-term conditions and frailty. Attempts to optimise glycaemic control should be undertaken, taking care to avoid hypoglycaemia in older or multi-morbid patients. Cardiovascular risk reduction with statins, lifestyle advice and smoking cessation should be strongly advocated, as well as regular screening for diabetes complications. eGFR should be measured at least 6 monthly.

Nephrological advice should be sought in patients with rapidly deteriorating renal function, rising ACR despite optimal treatment or eGFR $<30 \mathrm{~mL} / \mathrm{min} / 1.73 \mathrm{~m}^{2}$ when discussions about RRT will need to be undertaken.

\section{New treatments for DKD}

Over the past 2 decades, a number of interventions to limit the deterioration of renal function in DKD have shown promise, but have shown little benefit in randomised controlled trials (RCTs). These interventions include combined ACEI/ARB therapy, use of direct renin inhibitors, bardoxolone and endothelin-A receptor antagonists. ${ }^{6-9}$ In the last 5 years, however, cardiovascular outcomes trials (CVOTs) of newer agents used in T2D have led to important renal outcome data which now inform clinical practice.

\section{Sodium-glucose transporter-2 inhibitors}

Sodium-glucose transporter-2 inhibitors (SGLT-2i) act on the proximal renal tubule to induce glycosuria. They have a modest effect on improving glucose control, with a reduction of glycated haemoglobin $\left(\mathrm{HbA}_{1 \mathrm{c}}\right) \sim 10 \mathrm{mmol} / \mathrm{mol}(1 \%)$. They can improve body weight and blood pressure. Common adverse effects include genital mycotic infections or UTI. Less commonly, SLGT-2i may cause euglycaemic ketoacidosis, characterised by relative normoglycaemia and presence of ketoacidosis in a person with T2D. Careful patient education around sick day rules is needed (Box 1), including avoidance prior to surgery and avoidance of ketogenic diets. ${ }^{10}$

Several large RCTs suggest significant benefit of SGLT-2i drugs in patients with DKD independent of their effect on glycaemia. Most compelling of these studies was CREDENCE, which examined renal outcomes in patients treated with canagliflozin or placebo in 4,401 patients with T2D, CKD (mean eGFR $56.2 \mathrm{~mL} / \mathrm{min} / 1.73 \mathrm{~m}^{2}$ ) and albuminuria. ${ }^{11}$ Canagliflozin treatment was associated with a $34 \%$ reduction in the renal specific composite of doubling of baseline creatinine, ESRD or death from renal or cardiovascular causes (hazard ratio (HR) 0.66; 95\% confidence interval (CI) $0.53-0.81 ; p<0.001)$. Canagliflozin also reduced three-point major adverse cardiovascular outcomes (3p-MACE) by 20\% (HR 0.80; 95\% CI 0.67-0.95; $p<0.01)$ and hospitalisation for heart failure (hHF) by $39 \%$ (HR 0.61; 95\% CI 0.47-90.8; $p<0.001$ ). The numbers needed to treat (NNT) to prevent one renal outcome was 21.

Meta-analysis confirms favourable effects of SGLT-2i on the renal composite of doubling of serum creatinine (eGFR $40 \%$ decline), RRT initiation or renal death (risk reduction (RR) $0.63 ; 95 \%$ CI $0.56-0.71){ }^{12}$ Pooled NNT for renal outcomes was 67 . The effects appear to be strongest among those patients with albuminuria, and the effect is additive to ACEI or ARB use.
Box 1. Sick day rules when a person with diabetes is not well ${ }^{10}$

When a person with diabetes is not well or is unable to eat and drink as normal, some simple rules can help prevent further deterioration or development of diabetic ketoacidosis.

> Measure capillary ketones: using self-testing equipment or at the local hospital.

$>$ If $>1.5 \mathrm{mmol} / \mathrm{L}$, then attend the local emergency department immediately for testing for possible diabetic ketoacidosis.

> Take half glass of milk, fruit juice, yogurt or soups (not clear soups), if not able to eat and if taking insulin, cover with half the normal dose of insulin.

> Drink plenty of water / sugar free fluid to avoid dehydration for up to 24 hours.

$>$ Seek medical advice if infection or illness worsens.

$>$ Be aware that glucose levels can be normal because of the way sodium-glucose transporter- 2 inhibitors work. Ketone levels can be high, even with a normal glucose.

> Some medications are not good when you are not well. Diabetes Medicine to Stop Temporarily when not well (DAMN GlucoSe drugs; discuss with your doctor):

> diuretics: 'water pills' eg frusemide, bendroflumethiazide, indapamide or bumetanide

> angiotensin converting enzyme inhibitors: names ending in 'pril' eg ramipril, lisinopril or perindopril

> angiotensin receptor blockers: names ending in 'sartan' eg candesartan, losartan or irbesartan

$>$ metformin

$>$ non-steroidal anti-inflammatory drugs: anti-inflammatory pain killers eg ibuprofen, naproxen or diclofenac

> glucagon-like peptide-1 analogues (injectable): names ending in 'tide' eg exenetide, liraglutide, dulaglutide or semaglutide

> sodium-glucose transporter-2 inhibitors: names ending in 'flozin' eg canagliflozin, dapagliflozin, empagliflozin or ertugliflozin.

Based on this evidence, canagliflozin is now considered an effective option for renal and cardiovascular protection in DKD, and can be initiated in patients with T2D and macroalbuminuria, and $\mathrm{eGFR}>30 \mathrm{~mL} / \mathrm{min} / 1.73 \mathrm{~m}^{2}$ as add-on to ACEI, irrespective of glucose control. It can be continued below eGFR $30 \mathrm{~mL} / \mathrm{min} /$ $1.73 \mathrm{~m}^{2}$. It is expected that dapagliflozin will obtain a similar licence in due course as it has equally compelling data more recently published. ${ }^{13}$

In contrast to ACEI/ARBs, renoprotective effects of SGLT-2i are thought to be mediated by tubuloglomerular feedback, natriuresis and glucose induced osmotic diuresis which reduces intraglomerular pressure.

\section{Glucagon-like peptide-1 receptor agonists}

Glucagon-like peptide-1 receptor agonists (GLP-1RA) have a potent glucose lowering effect by mimicking the effect of incretin hormones in stimulating insulin release, reducing glucagon and reducing appetite. They aid significant weight reduction. Adverse effects are predominantly gastrointestinal disturbance and, more rarely, pancreatitis. GLP-1RAs have shown promising results in CVOTs. Meta-analysis of 56,004 patients showed a $12 \%$ reduction 
in 3p-MACE in GLP-1RA treated patients. ${ }^{14} \mathrm{~A}$ composite renal outcome was reduced by $17 \%$ for all GLP1-RA, but this is mainly due to a reduction in new onset macroalbuminuria, rather than a reduction in progressive renal disease, ESRD or renal-related death. Liraglutide, dulaglutide and semaglutide have demonstrated effective glycaemic control in T2D and CKD and are licensed for use down to eGFR $15 \mathrm{~mL} / \mathrm{min} / 1.73 \mathrm{~m}^{2}$.

\section{Aldosterone receptor antagonist}

A study of the aldosterone receptor antagonist, Finerenone in 5,734 patients with T2D and CKD showed some positive outcomes. ${ }^{15}$ ESRD, sustained decrease of $>40 \%$ in eGFR or death from renal causes was reduced by $18 \%$ in the Finerenone group (HR 0.82; 95\% CI 0.73-0.93; $p=0.001$ ). Hyperkalaemia due to Finerenone occurred in $2.3 \%$ of patients treated.

\section{Recent guidelines on management of DKD}

Current UK guidance based on the National Institute for Health and Care Excellence (NICE) has not been updated since 2015. More recently, updated UK guidelines from the Association of British Clinical Diabetologists (ABCD) and The Renal Association (RA) advocate SGLT-2i in all patients with T2D and CKD or albuminuria irrespective of glucose control. ${ }^{16}$ Furthermore, guidance from the American Diabetes Association and European Association for the Study of Diabetes (ADA-EASD) recommend that SGLT-2i should be used in any patient with T2D with heart failure or $\mathrm{CKD}{ }^{17}$

\section{Diabetes on dialysis}

Diabetes is common among patients on haemodialysis (HD) and managing glucose control in people with diabetes on HD can be challenging. Dialysis improves uraemia, acidosis and hyperphosphataemia which can periodically improve insulin secretion, leading to risk of hypoglycaemia, and symptoms of hypoglycaemia may be confused with hypotension. Furthermore, assessment of glucose control may be tricky due to difficulties in interpretation of $\mathrm{HbA}_{1 \mathrm{c}}$ in renal anaemia. Glycaemic management is therefore reliant on frequent self-monitoring of blood glucose (SMBG) which is burdensome. In addition, a number of antihyperglycaemic agents (such as metformin, SGLT-2i and GLP1RA) cannot be used in dialysis patients, further adding to the challenge.

Guidance on the management of diabetes in patients on HD has been published and is due to be updated. ${ }^{18}$ There is some evidence that asymptomatic hypoglycaemia is common among patients on $\mathrm{HD}$, and therefore updated guidance is likely to suggest that use of continuous or flash glucose monitoring (CGM or FGM) technology may help in the management of patients with diabetes on HD (Fig 1). Intermittent 'diagnostic' use of CGM or FGM in patients on insulin or sulphonylurea on HD. NHS guidance on the use of the FGM (Freestyle Libre) includes any form of diabetes on HD on insulin.

Management of glycaemia in patients on peritoneal dialysis can be even more challenging especially when high glucose dialysate fluids are used. This can lead to a vicious cycle of hyperglycaemia, increased fluid intake and fluid overload, necessitating higher dialysate concentrations. CGM or FGM may be useful to gain a clear understanding of glucose variability with dialysate bags to enable adjustment of insulin doses on dialysis and non-dialysis days.

\section{Post-transplant diabetes mellitus}

Advances in immunosuppression have enabled dramatic improvements in outcomes of solid organ transplantation (SOT), with major improvements in graft and patient survival. Morbidity and mortality from cardiovascular disease is, however, increasing, and diabetes is a major contributor. Diabetes may pre-date transplantation, but is also a common consequence of transplantation, affecting between $10 \%-40 \%$ of SOT recipients. Post-transplant diabetes mellitus (PTDM) confers a significantly higher risk of graft failure and mortality. ${ }^{19}$ Guidance on the diagnosis, management and prevention of PTDM have been developed by the $A B C D$ and RA. ${ }^{20}$

\section{Pathogenesis}

Weight gain (due to glucocorticoids and fewer dietary restrictions) is common in patients post-SOT. While excess weight, ethnicity and sedentary lifestyles are all risk factors for development of PTDM, other risk factors include immunosuppression (especially calcineurin inhibitors (CNIs)) and infection (eg hepatitis C). Tacrolimus is a highly effective immunosuppressant but has a potent adverse effect on beta-cell function, leading more frequently to significant hyperglycaemia compared with ciclosporin.

\section{Diagnosis}

Early hyperglycaemia is ubiquitous in SOT recipients due to stress, infection and immunosuppression. In the immediate posttransplant period, screening for post-transplant hyperglycaemia should involve frequent capillary blood glucose (CBG) testing, mainly later in the day (post-lunch or evening meal). A subsequent diagnosis of PTDM should only be confirmed by oral glucose tolerance test or $\mathrm{HbA}_{1 \mathrm{c}}$ at least 3 months post-transplant, when immunosuppression is stabilised.

\section{Management}

Early post-transplant hyperglycaemia requires active monitoring and management (Fig 1). Mild hyperglycaemia may respond to oral hypoglycaemic agents, but $C B G s>14 \mathrm{mmol} / \mathrm{L}$ require intravenous or subcutaneous insulin. Subsequent management should mirror that of T2D pathways. Concern about the risk of urinary infection with SGLT-2i agents has limited their use in this condition, but their reno-protective effects may be very useful in PTDM, and small studies suggest no increase in risk of infection. Outcomes of larger studies are awaited. Change in immunosuppression regimen may aid management of hyperglycaemia and, if feasible, consideration may be given for conversion of tacrolimus to other immunosuppressive regimens in patients with difficult hyperglycaemia.

PTDM may be most effectively managed in a multidisciplinary setting with diabetes and transplant specialists.

\section{Conclusion}

Management of early DKD using SGLT-2i irrespective of glycaemic control is highly evidence based and recommended by a number of guidelines. This evidence needs to be implemented as it is likely to be highly cost effective to prevent progression to ESRD. 
Pre-operatively

- Establish risk factors for diabetes (obesity family history previous GDM, high-risk ethnic group or glucocorticoid therapy). - Monitor FPG and HbA1c, ideally 6 monthly (high risk if FPG 6.1-6.9 mmol/L or $\mathrm{HbA}{ }_{1 \mathrm{c}} 42-47 \mathrm{mmol} / \mathrm{mol}(6.1 \%-6.4 \%)$ ).

- If high risk for diabetes, advise weight loss, increased exercise and improved diet, and smoking cessation.

- If $\mathrm{HbA} \mathrm{Al}_{\mathrm{c}} \geq 48 \mathrm{mmol} / \mathrm{mol}$ or FPG $\geq 7.0 \mathrm{mmol} / \mathrm{L}$ on two occasions, diagnose diabetes and put on to standard diabetes pathway.

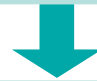

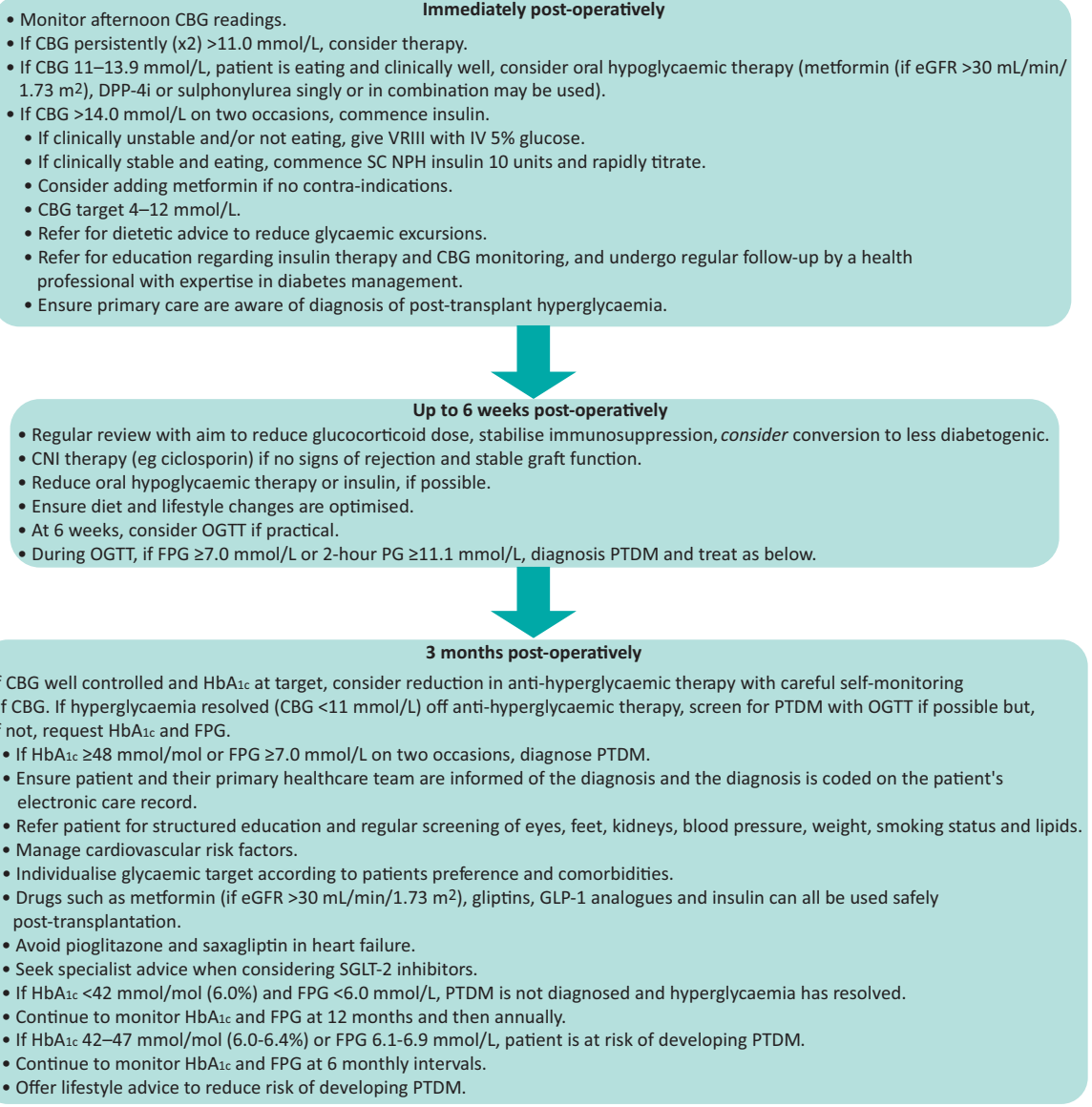

Fig 1. Pathway for diagnosis and management of post-transplant diabetes mellitus. 2-hour PG = 2-hour plasma glucose; $C B G=$ capillary blood glucose; $\mathrm{CNI}=$ calcineurin inhibitor; DPP-4i = dipeptidylpeptidase-4 inhibitor; eGFR = estimated glomerular filtration rate; FPG = fasting plasma glucose; GDM = gestational diabetes mellitus; $\mathrm{HbA}_{1 \mathrm{c}}=$ glycated haemoglobin; IV = intravenous; $\mathrm{NPH}=$ neutral protamine Hagedorn; PTDM = post-transplant diabetes mellitus; OGTT = oral glucose tolerance test; $\mathrm{SC}=$ subcutaneous; SGLT-2 = sodium-glucose transporter-2; VRIII = variable rate intravenous infusion of insulin.
Patients with diabetes on RRT (dialysis or transplantation) may have particular challenges and require multidisciplinary management between diabetes and renal specialists. New guidelines are available to help manage these complex and vulnerable patients.

\section{References}

1 International Diabetes Federation. Diabetes facts and figures. IDF, 2020. www.idf.org/aboutdiabetes/what-is-diabetes/facts-figures. html [Accessed 17 February 2021].

2 Kainz A, Hronsky M, Stel VS et al. Prediction of prevalence of chronic kidney disease in diabetic patients in countries of the European Union up to 2025. Nephrol Dial Transplant 2015; 30(Suppl 4):113-8.

3 Koye DN, Shaw JE, Reid CM et al. Incidence of chronic kidney disease among people with diabetes: a systematic review of observational studies. Diabet Med 2017;34;887-901.

4 Fox CS, Matsushita K, Woodward M et al. Associations of kidney disease measures with mortality and end-stage renal disease in individuals with and without diabetes: a meta-analysis. Lancet 2012:380:1662-73.
5 Sugahara M, Pak WLW, Tanaka T, Tang SCW, Nangaku M. Update on diagnosis, pathophysiology, and management of diabetic kidney disease. Nephrology (Carlton) 2021;26:491-500.

6 Fried L, Emanuele N, Zhang ] et al. Combined Angiotensin Inhibition for the Treatment of Diabetic Nephropathy. N Engl J Med 2013;369: 1892-903.

7 Parving H, Brenner B, McMurray ] et al. Cardio renal end points in a trial of aliskiren for type 2 diabetes. N Engl J Med 2012;367: 2204-13.

8 Zeeuw D, Akizawa T, Audhya P et al. Bardoxolone methyl in type 2 diabetes and stage 4 chronic kidney disease. $N$ Engl J Med 2013;369:2492-503.

9 Mann J, Green D, Jamerson K et al. Avosentan for overt diabetic nephropathy. JASN 2010;21:527-35.

10 Dashora U, Patel D, Nagi D et al. SGLT-2 inhibitor in people with type 2 diabetes: an educational resource for health professionals. Association of British Clinical Diabetologists. https://abcd.care/ sites/abcd.care/files/site_uploads/Resources/Position-Papers/SGLT2inhibitors-ABCD.pdf [Accessed 17 February 2021].

11 Perkovic V, Jardine MJ, Neal B et al. Canagliflozin and renal outcomes in type 2 diabetes and nephropathy. N Engl J Med 2019;380: 2295-306. 
12 Lo KB, Gul F, Ram P et al. The effects of SGLT2 inhibitors on cardiovascular and renal outcomes in diabetic patients: a systematic review and meta-analysis. Cardiorenal Med 2020;10:1-10.

13 Heerspink HJL, Stefánsson BV, Correa-Rotter R et al. Dapagliflozin in patients with chronic kidney disease. N Engl J Med 2020;383: 1436-46.

14 Kristensen SL, Rørth R, Jhund PS et al. Cardiovascular, mortality, and kidney outcomes with GLP-1 receptor agonists in patients with type 2 diabetes: a systematic review and meta-analysis of cardiovascular outcome trials. Lancet Diabetes Endocrinol 2019;7:776-85.

15 Bakris GL, Agarwal R, Anker SD et al. Effect of finerenone on chronic kidney disease outcomes in type 2 diabetes. N Engl J Med 2020;383:2219-29.

16 Association of British Clinical Diabetologists, The Renal Association. Clinical practice guidelines for management of hyperglycaemia in adults with diabetic kidney disease: 2021 update. ABCD, 2021. https://renal.org/sites/renal.org/files/Managing \% 20hyperglycaemia \% 20in \% 20people \% 20with \% 20DKD_final \% 20draft.pdf [Accessed 05 May 2021].

17 Buse JB, Wexler DJ, Tsapas A et al. 2019 Update to: Management of hyperglycemia in type 2 diabetes, 2018. A consensus report by the American Diabetes Association (ADA) and the European Association for the Study of Diabetes (EASD). Diabetes Care 2020:43:487-93.
18 Frankel A, Kazempour-Ardebili S, Bedi R et al. Management of adults with diabetes on the haemodialysis unit: summary of new guidance from the Joint British Diabetes Societies and Renal Association. Diabetic Medicine 2018;35:1018-26.

19 Kuo HT, Sampaio MS, Vincenti F et al. Associations of pretransplant diabetes mellitus, new-onset diabetes after transplant, and acute rejection with transplant outcomes: an analysis of the Organ Procurement and Transplant Network/United Network for Organ Sharing (OPTN/UNOS) database. Am J Kidney Dis 2010;56:1127-39.

20 Chowdhury TA, Wahba M, Mallik R et al. Association of British Clinical Diabetologists and Renal Association guidelines on the detection and management of diabetes post solid organ transplantation. Diabet Med 2021;38:e14523.

Address for correspondence: Dr Tahseen A Chowdhury, 7th Floor, John Harrison House, The Royal London Hospital, Whitechapel, London E1 1BB, UK.

Email: tahseen.chowdhury@nhs.net 